\title{
Acciones de superación profesional para potenciar la competencia en comunicación de resultados científicos
}

\author{
Actions for Professional Development to Enhance Competence in Communicating Scientific Results
}

\author{
Alexeis Ruíz-Díaz \\ Centro Universitario Municipal de Sagua la Grande \\ Sagua la Grande, Cuba \\ alex6_ruiz@hotmail.com \\ Yamila Roque-Doval \\ Universidad Central "Marta Abreu" de Las Villas, \\ Centro de Estudios Comunitarios, \\ Santa Clara, Cuba \\ yamilar@uclv.edu.cu \\ Mayra Rodríguez-Ruíz ${ }^{3}$ \\ Universidad Central "Marta Abreu" de Las Villas \\ Santa Clara, Cuba \\ mayrar@uclv.edu.cu
}

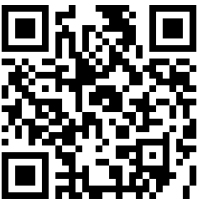

Recibido 28 de octubre de 2015 • Corregido 30 de noviembre de 2016 • Aceptado 10 de abril de 2017

\begin{abstract}
'Licenciado en Comunicación Social, especialidad de Periodismo, por la Universidad de La Habana, Cuba. Es Máster en Educación Superior, mención Docencia Universitaria, porla UCLV, Cubay Doctor en Ciencias Pedagógicas por la Universidad Central "Marta Abreu" de Las Villas (UCLV), Cuba. Es profesor auxiliar y labora en el Centro Universitario Municipal (CUM) de Sagua la Grande, perteneciente a la Universidad Central "Marta Abreu” de Las Villas. También ha impartido clases en el Centro de Estudios de la Radio y la Televisión (CERT). Se ha especializado en disciplinas como teoría de la comunicación y sociolingüística. Su línea investigativa contempla las áreas temáticas de la comunicación para el desarrolloy la competencia comunicativa. De manera simultánea trabaja como director y guionista de programas informativos de televisión.

${ }^{2}$ Licenciada en Psicología, por la Universidad Central de Las Villas. Es Máster en Ciencias de la Educación por esta misma universidad cubana y Doctora en Ciencias de la Educación, por la Universidad Central "Marta Abreu" de Las Villas (UCLV), Cuba. Es profesora titular y labora en el Centro de Estudios Comunitarios, adscrito a la Facultad de Ciencias Sociales de la UCLV, Cuba. Se ha especializado en disciplinas como la psicología social. Lidera el proyecto investigativo de Villa Clara sobre envejecimiento poblacional, así como otros sobre el impacto del desarrollo comunitario en la provincia. Es la vicepresidenta del Consejo de las Ciencias Sociales en Villa Clara.

\footnotetext{
${ }^{3}$ Licenciada en Lengua Inglesa, por la Universidad de La Habana. Es Máster en Lengua Inglesa, por la Universidad Central "Marta Abreu" de Las Villas (UCLV) y Doctora en Pedagogía por esta misma universidad cubana. Es profesora titular e imparte docencia en la carrera de Lengua y Literatura Inglesa en la Facultad de Humanidades de la UCLV. Desde hace varios años es presidenta del Tribunal de Exámenes de Candidatura en Idioma Inglés en esta casa de altos estudios. Coordina la Maestría en Estudios de Lengua Inglesa, actualmente en su tercera edición. Su línea investigativa se inclina hacia las estrategias de aprendizaje en la enseñanza de la lengua inglesa.
} 
doi: http://dx.doi.org/10.15359/ree.21-2.2

URL: http://www.una.ac.cr/educare

CORREO: educare@una.cr

Resumen: El objetivo general de este artículo de investigación es proponer un plan de acciones de superación profesional que contribuya a potenciar la competencia en comunicación de resultados científicos en docentes de los centros universitarios municipales. Se parte de un enfoque de complementariedad metodológica que combina diferentes métodos cuantitativos y cualitativos en función de objetivos específicos. El contexto de intervención y diagnóstico es el Centro Universitario Municipal de Sagua la Grande y la muestra la constituyó el total de 25 profesoras y profesores que conforman el claustro de la carrera Ingeniería Industrial de esta institución, adscrita a la Universidad Central "Marta Abreu" de Las Villas, en Cuba. Se ofrecen las regularidades emanadas de la etapa de diagnóstico inicial de las necesidades de superación del personal docente muestreado y del nivel en que se encontraba la variable competencia en comunicación de resultados científicos. Con esa información que justifica los propósitos de la investigación, se modela el proceso de superación con enfoque de sistema y se ofrece como resultado práctico la organización y diseño de un plan de acciones de superación a través de formas de postgrado como el diplomado y el entrenamiento para la fase de implementación.

Palabras clave: Superación profesional; competencia comunicativa; comunicación científica; desarrollo local.

\begin{abstract}
The main goal of this research is to propose an action plan for professional development to enhance competence in communicating scientific results in teachers of municipal secondary schools. The research is part of a complementary methodological approach that combines different quantitative and qualitative methods based on specific objectives. The diagnosis was made at the Municipal University Center of Sagua La Grande, with a sample of 25 teachers who are members of the Industrial Engineering career staff at the institution, attached to the Central University "Marta Abreu" of Las Villas, Cuba. Regularities were found from the stage of the initial diagnosis of professional development needs and the level in which the "competence in communicating scientific results" variable was. With this information justifying the purposes of this research, a process was designed following a systemic approach, and the practical result was the proposal of the organization and design of an action plan for development through postgraduate education forms during the implementation phase.
\end{abstract}

Keywords: Professional development; communicative competence; scientific communication; local development.

\title{
Introducción
}

La superación profesional del personal docente universitario es una expresión de su formación continua orientada al perfeccionamiento de sus modos de actuación en el escenario educativo. En el contexto de la educación de postgrado en Cuba se implementa esta, a través de varias formas, entre las que se destaca la concepción de superación profesional dentro de los Estudios de Avanzada, que concibe ese perfeccionamiento de su actividad pedagógica profesional como un mejoramiento humano. 
Con este artículo se desea compartir la experiencia del diseño de una propuesta de acciones de superación profesional con una concepción sistémica del proceso para contribuir a solucionar la problemática de la insuficiente actividad científica del profesorado, dada por la escasa comunicación de resultados científicos, sobre todo en cuanto a publicaciones en revistas científicas y la participación en eventos científicos institucionales de primer nivel.

La pertinencia de este artículo de investigación radica en que la comunicación científica suele abordarse solamente desde la categoría "habilidad", sin embargo, las acciones de superación propuestas aquí se orientan en un proceso de transformación del estado de la competencia en comunicación de resultados científicos en el personal docente de los centros universitarios municipales y ello se garantiza con una didáctica integradora a través de métodos de estímulo a la actividad productiva.

Actualmente el profesorado universitario está llamado no solo a ser mediador entre el conocimiento establecido y su alumnado ávido de ese conocimiento. Su integralidad profesional les lleva a participar activamente en la actualización, contextualización y hasta la generación de nuevos conocimientos, estimulando en sus estudiantes el carácter transformador de la realidad y de sí que es sinónimo del aprovechamiento creativo de ese conocimiento: saber-saber, hacer-saber, ser. Incluso, esta tríada puede crecerse en otras dimensiones y ya se llegaría a terrenos más complejos que el domino de conocimientos, habilidades y destrezas como tradicionalmente suele verse.

En la integralidad consustancial al desempeño profesional del personal docente está el mantener en justo equilibrio la dimensión investigativa, que a su vez les convertirían en profesoras y profesores más preparados para enfrentar creativa y de manera transformadora la misión de formar a la sucesiva generación científica del país, aunque también tengan metas más cercanas e institucionales como ser garantes participativos y participativas en los procesos sobre los cuales la universidad ofrece evidencias de calidad.

Autoridades del Ministerio de Educación Superior en Cuba valoran la importancia de la gestión del conocimiento dentro de los procesos universitarios y, a su vez, lo relacional con el desarrollo social:

Las nuevas necesidades provocadas por el actual contexto económico, social y tecnológico, la aplicación de la gestión del conocimiento debe encaminarse tanto en la reorganización interna de procesos, como en la mejora de la docencia y la investigación, con el objetivo de facilitar el desarrollo de una universidad competitiva y adaptada a las nuevas demandas de la sociedad. (Estrada y Benítez, 2006, p. 3)

Precisamente una estrategia encaminada en la educación superior cubana ha sido la universalización de esta enseñanza, que ha tenido una experiencia muy contextualizada, con más de una década, conocida como la municipalización. Hoy ello se traduce en los centros universitarios 
doi: http://dx.doi.org/10.15359/ree.21-2.2

URL: http://www.una.ac.cr/educare

CORREO: educare@una.cr

municipales (CUM) que, a su vez, hacen realidad otras estrategias concomitantes en el vínculo universidad-sociedad, y cabe mencionar aquí su relación como entidad asesora de la Iniciativa de Desarrollo Local, implementada en cada territorio por el Ministerio de Economía y Planificación; también la superación de cuadros, y otras potencialidades que el Ministerio de Educación Superior le confía como centro generador de conocimientos y actividad científica en pos del desarrollo.

Estudios cubanos del vínculo entre las universidades y el desarrollo social han insistido en que la utilización oportuna y sistemática del conocimiento científico aportado por las investigaciones sociales resulta muy necesaria para el desarrollo sostenible, especialmente desde la dimensión local; pero se carece de una efectiva política de gestión del conocimiento -incluidos los procesos comunicativos de socializar dichos conocimientos- que se integre a la estrategia de desarrollo de los territorios y que establezca indicadores para valorar la apropiación social del conocimiento y en qué medida este contribuye a alcanzar las metas sociales deseables (Núñez, Montalvo y Pérez, 2006).

Señalan Ruíz y Roque (2015) que dentro de la "gestión universitaria del conocimiento científico para el desarrollo'existe un eslabón deficiente y es la comunicación de esos resultados científicos que completan la investigación tanto en las fases de aplicación como los resultados posteriores de impacto en la sociedad, y que a su vez contrastan con lo que conocemos como producción científica satisfactoria"' (p. 88).

Interesa en este trabajo mostrar una contribución que aminore tal incongruencia desde una perspectiva que implique la respuesta responsable, eficiente y exitosa de investigadoras e investigadores, y en este caso docentes de los centros universitarios municipales -en lo adelante CUM- que, en aras de su formación permanente, deben potenciar su esfera de actuación investigativa.

Con este antecedente, se declara como objetivo general de este trabajo proponer un plan de acciones de superación profesional que contribuya a potenciar la competencia en comunicación de resultados científicos en las docentes y los docentes de los centros universitarios municipales.

A su vez, el conjunto de actividades que encaminan tal propósito tiene como finalidad crear una base de conocimientos teóricos, prácticos y metodológicos, complementados por otros aspectos afectivo-motivacionales y procedimentales que permitan un exitoso desempeño comunicativo de la actividad científica profesional en contextos académicos, docentes y sociales en general, a partir del dominio integrado de recursos comunicativos y personológicos en docentes-estudiantes tomando como fundamento las experiencias cubanas en la formación de competencias. 


\section{Breve referencia teórica}

La estrategia de la universalización de la educación superior en Cuba, desde el precepto de Educación para todos pronunciado por la UNESCO fundamenta lo que se conoce como Nueva Universidad Cubana, sobre todo por encaminar experiencias concretas como la "municipalización". Los Centros Universitarios Municipales constituyen el resultado institucional y organizativo de esta experiencia que ahora ha orientado sus procesos sustantivos en la llamada Iniciativa para el Desarrollo Local, rectorada por los gobiernos municipales a instancias del Ministerio de Economía y Planificación en Cuba. En esto, la universidad de localidad, como le llaman, aporta su mayor riqueza que es el conocimiento y su capital social, en este caso sus profesores y profesoras.

Lo anterior también está contenido en la llamada agenda priorizada educación superiordesarrollo local y es el resultado de asumir teórica y sociológicamente los conceptos de desarrollo social, desde el llamado modelo del contexto que "configuran una epistemología diferente del conocimiento al subrayar que la producción, distribución y uso de los conocimientos y tecnologías tienen lugar siempre en contextos particulares, con sus singularidades económicas, culturales, valorativas, y que ellos deben modelar sus prioridades y desarrollo" (Souza, 2001, citado por Núñez, 2010, p. 198).

Esos grupos profesionales de la educación superior que producen los conocimientos actúan con responsabilidad, compromiso y éxito en la solución de problemas del contexto y así lo transforman y se transforman a sí mismos. Para lograr esa calidad en el desempeño, es oportuno un proceso de formación y existen varios enfoques formativos basados en competencias desde el pasado siglo XX, de lo cual en esta investigación dan fe Abarca-Fernández (2010); Escudero (2008); Posada (2004); Ruiz (2010); Tobón (2005); Zabalza (2012); entre otros.

Castellanos, Fernández, Llivina, Arencibia y Hernández (2003); González y Ramírez (2011); González (2006), Pla (2002), Tejeda y Sánchez (2012) se refieren a la formación de profesionales competentes en el contexto educativo cubano, con una concepción humanista de la competencia y dentro de ella un enfoque integrador-contextualista consistente en ver al constructo como unidades integradas y complejas (las configuraciones psicológicas) que no solo se manifiestan en el desempeño eficiente de cada individuo, sino que también conciben toda la movilización que hace de sus recursos psicológicos y personológicos como determinantes de esa actuación exitosa en un contexto (Mesa, 2011).

Interesa a esta investigación recorrer el camino teórico de la competencia comunicativa que, con su función mediadora de las relaciones sociales, sigue el propósito de crear e intercambiar significados entre los sujetos que participan del acto comunicativo. Vista como una competencia profesional, entre tantas clasificaciones, no se obvia el camino común entre lengua-contexto verbal y situacional que, luego de Noam Chomsky, abrió D. Hymes a finales 
doi: http://dx.doi.org/10.15359/ree.21-2.2

URL: http://www.una.ac.cr/educare

CORREO: educare@una.cr

de la década de 1960 y toda su evolución hasta el presente que ha llevado a la competencia comunicativa a otros escenarios profesionales, y ahí cuentan estudios latinoamericanos como los de Beltrán (s. f.); Chan (2000); De la Rosa (2004); Tobón (2005), por citar los fundamentales.

El término en Cuba ha sido muy tratado en el plano de la didáctica de la lengua, sobre todo la enseñanza de idiomas, aunque también se ha aprovechado en otros contextos profesionales específicos; sinembargo, es tendencia predominante sutratamientolingüísticoque parte del enfoque cognitivo, comunicativo y sociocultural de Roméu (2007). Esto se aprecia en las investigaciones de Concepción (2010); De la Paz (2011); Del castillo y Rodríguez (2015); Mariño y Ortiz (2011); Mesa (2011); Pompa y Pérez (2015); Recino, Quesada, Finalet y González (2014); entre otras.

A partir de una exhaustiva revisión bibliográfica, no le consta a la autoría de esta investigación que exista una definición para la competencia en comunicación de resultados científicos con toda la integración de otros términos que propone denotativamente; pero no se desestima que existen investigaciones que se refieren a la actividad de la comunicación de resultados científicos desde la estructura psíquica de la habilidad y siguiendo el paradigma formal comunicativo que se concentra en las tipologías discursivas para la publicación en revistas científicas, o sea, la producción bibliográfica.

Una visión comunicológica del proceso comunicativo contraria al enfoque difusivo que habla de intercambio de información direccional entre un sujeto emisor y un sujeto receptor, se decanta por el enfoque expresivo que entiende a la comunicación como un "hacer común", es decir, con una interacción social democrática, de negociación, reconocimiento mutuo, comunicación en el compartir, en la participación (Freire, 2004; Martín-Barbero, 2003). Así los conceptos de competencia comunicativa y competencia en la comunicación de resultados científicos, relacionados en esta investigación, se inscriben dentro del enfoque integralcontextualista o configuracional psicológico, como también se le conoce en Cuba y que tiene su base en la personalidad del sujeto.

La superación como vía para el mejoramiento profesional es otro de los presupuestos teóricos en esta investigación que cuenta con los fundamentos aportados por Añorga-Morales (2014); Concepción (2010); Fraga (2005); García-Ribeaux y Zaldívar (2013); González y Alonso (2012); Mesa (2011); Peralta (2013), Valdesprieto y Delgado(2012); entre otros.

Son muchas las maneras de abordar este componente de la llamada "cuarta enseñanza", aunque a veces difieren el avance teórico que fundamenta el valor transformador de la superación y la realidad de la práctica educativa donde imperan estilos tradicionales y conservadores. Asimismo, es poco el tratamiento interdisciplinario y transdisciplinario en los contenidos formativos propuestos ni se aprovechan las verdaderas potencialidades que desde lo metodológico contienen las formas del postgrado. 
También la superación profesional en el ámbito de la educación superior enfatiza en el ejercicio de las funciones pedagógicas, por ello se busca la mejora de la actuación profesional de cada docente en el aula y pocas veces hacia la actualización de su propia ciencia o perfeccionar su desempeño en otras facetas complementarias como lo investigativo proyectado hacia una dimensión social más amplia.

La presente investigación asume el concepto sistematizado de superación profesional en docentes de la SUM ${ }^{4}$, de González y Alonso (2012) y que ha definido como:

Proceso docente -educativo de carácter continuo, planificado por y para el docente que labora a tiempo parcial o completo en la SUM [sic] con el propósito de actualizar y perfeccionar su desempeño profesional pedagógico actual y/o perspectivo en correspondencia con los requerimientos de la universidad cubana actual. (p. 5)

Según los autores, sus indicadores de medición son:

- Grado de correspondencia entre la planificación de la superación y la demanda.

- Grado de correspondencia entre los problemas a resolver y los objetivos de la superación ofertada.

- Grado de satisfacción de docentes y alumnos.

- Grado de satisfacción de los funcionarios de las estructuras de dirección.

Para el trabajo operacional se asume en esta investigación que la definición de competencia en comunicación de resultados científicos es a su vez el resultado de un complejo proceso de construcción teórica de un concepto y en su representación verbal se entiende como una configuración psicológica compleja que en unidad cognitiva, afectivomotivacional y procedimental se autorregula en el sujeto para su desempeño eficiente, al compartir resultados de su actividad científica y adecuarse a las exigencias del contexto comunicativo a través del conocimiento y manejo de recursos lingüísticos, textuales, pragmáticos, tecnológicos y socioculturales.

Los recursos comunicativos se aprecian en los contenidos de cada dimensión de la variable de trabajo y específicamente en los indicadores que la operacionalizan.

${ }^{4}$ La investigación que se toma como referente y en el año de su realización alude a sede universitaria municipal (SUM), primera de las denominaciones que tuvieron estos centros docentes antes de la actual organización y estrategia de integración proyectada por el Ministerio de Educación Superior. 
doi: http://dx.doi.org/10.15359/ree.21-2.2

URL: http://www.una.ac.cr/educare

CORREO: educare@una.cr

La variable de trabajo se refiere a una actividad de la comunicación con un enfoque de competencia que tiene su base funcional en estructuras de la personalidad, por eso se coincide con Mesa (2011) y Ortiz (2006) y cuando amplían las dimensiones desde sus tipologías tradicionales de procesos psíquicos de la personalidad a terminologías más conjugadas con el contenido de la actividad. Basado en lo anterior, se utilizan las dimensiones e indicadores establecidos por Ruíz y Roque (2015):

Dimensión cognitiva: Definida por aquellos procesos psíquicos que le permitan al sujeto conocer la realidad natural y social a partir del reflejo que hagan de ella y que en este caso incluyen componentes como "conocimientos"; "habilidades (conocimientos en acción que ejercen una regulación ejecutora)"; "capacidades (con su carácter de potencialidad y un contenido más complejo)".

- Conocimientos de su ciencia.

- Conocimientos sobre la construcción de textos científicos.

- Habilidades expresivas e interpretativas de mensajes.

- Conocimientos para el uso de las tipologías discursivas del texto científico.

- Conocimiento de las normas éticas para la actividad científica.

- Conocimientos para el uso de las tecnologías en función de la comunicación de resultados científicos.

- Capacidad de autorreflexión sobre su adquisición, uso y desarrollo de esos conocimientos, habilidades y capacidades.

Dimensión afectivo/motivacional:Definida por los procesos psíquicos dela personalidad que movilizan desde sus componentes hacia la actividad y determinan el desempeño, e incluyen "sentimientos"; "necesidades"; "motivos"; "actitudes"; "propósitos"; "intereses"; "expectativas".

- Motivación por compartir (expresar/socializar) los resultados de su actividad científica.

- Interés por el conocimiento y los resultados científicos de otros.

- Motivación por cumplir los requerimientos éticos de la actividad científica.

- Expectativa positiva hacia el éxito de la actividad.

- Valoración adecuada del entorno comunicativo para conducirse con empatía y tolerancia. 
Dimensión procedimental: Definida por la expresión consciente y organizada de dominio de la acción y que tiene su base funcional de desempeño comunicativo en la unidad cognitivo-afectiva.

- Destrezas en la planificación, organización y ejecución de actividades de comunicación de resultados científicos.

- Destrezas en la gestión de publicaciones y participación en eventos.

- Destrezas en el manejo estratégico y direccional de la comunicación.

- Comportamiento ético y responsable en su actividad científica.

- Destreza en la selección, acceso y operación de las herramientas tecnológicas en función de la comunicación de resultados científicos.

- Disposición para la creación de un ambiente asertivo de intercambio en la comunidad.

Es necesario apuntar que la amplitud de indicadores no es sinónimo de trabajarlos indistintamente, pues al estar interrelacionados en una compleja actividad se trabajan por integraciones. La operacionalización de la variable de trabajo tuvo una valoración promedio de MUY ADECUADA por 15 profesoras y profesores expertos del ámbito de la educación superior, la totalidad con la categoría científica de Doctor en Ciencias Pedagógicas.

\section{Metodología empleada}

En este trabajo se defiende el diseño y organización de un plan de acciones de superación con una concepción sistémica de todo el proceso (García-Ribeaux y Zaldívar, 2013). Basado en lo anterior se ofrece la siguiente organización estructural sistémica:

- Entrada o fase in put (1):

- Competencia del profesor o profesora para comunicar resultados científicos.

- Fase pre-activa (2):

- Diagnóstico de necesidades de superación.

- Requisitos psicopedagógicos y componentes personológicos de participantes.

- Diseño del sistema de acciones de superación (Planeación y organización).

- Fase activa o interactiva (3)

- Implementación de las acciones de superación.

- Fase post-activa (4):

- Evaluación del impacto inmediato del proceso de superación. 
doi: http://dx.doi.org/10.15359/ree.21-2.2

URL: http://www.una.ac.cr/educare

CORREO: educare@una.cr

Entrada o fase in put (1): El contexto para la implementación es el municipio de Sagua la Grande, en la provincia de Villa Clara. Se trata de un territorio de carácter industrializado, con una fuerte actividad científica y considerado el segundo en importancia, luego de la capital Santa Clara. Allí existe el Centro Universitario Municipal "Mario Rodríguez Alemán" conformado por tres antiguas filiales de educación superior, la propia de la Universidad Central de Las Villas, la de la Universidad Pedagógica y la de la Universidad de la Cultura Física y el Deporte.

La muestra ha tomado los 25 profesoras y profesores de la carrera Ingeniería Industrial, con fundamento en que es el claustro más estable del centro y que reúne las dos modalidades de contratación ilustrativas de estos centros universitarios: la modalidad a tiempo completo y a tiempo parcial. Además, es la única carrera que tiene todos los años en formación, desde primer año hasta sexto año.

Para el diagnóstico de necesidades de superación y estado inicial de la competencia en comunicación de resultados científicos se emplearon métodos como el análisis de documentos, la observación directa, la entrevista a personal directivo de diferentes niveles (sede central y del Centro Universitario Municipal de Sagua la Grande), entrevista a informantes clave, entrevista grupal a estudiantes y a docentes, encuesta a profesorado. Luego, a través de la técnica de triangulación de fuentes y metodológica de los resultados de cada método aplicado, se arribó a las siguientes regularidades sobre el estado inicial de la competencia en comunicación de resultados científicos en las profesoras y profesores de la carrera Ingeniería Industrial del CUM de Sagua la Grande:

\section{Desde la dimensión cognitiva:}

1. Existe un conocimiento MEDIO sobre los componentes de la actividad científica y el basamento teórico que fundamenta la investigación como dimensión indispensable para el desempeño integral y exitoso de un profesional y particularmente de la educación superior.

2. Es MEDIO el conocimiento de las tipologías de la comunicación científica, y es MEDIO el dominio de habilidades lingüísticas, textuales, pragmáticas, tecnológicas y socioculturales que ponen esos conocimientos en acción, al igual que es MEDIA la potencialidad de su capacidad metacognitiva.

\section{Desde la dimensión afectivo-motivacional:}

3. Existe una motivación MEDIA del personal docente por la actividad científicoinvestigativa que lleva a justificar su poco conocimiento y deficiente desempeño en la comunicación de resultados científicos, al igual que resulta MEDIA su valoración de la actividad como indispensable en el desempeño integral, sin embargo, existe un sentimiento positivo hacia lo colaborativo de la otra persona en un proceso de construcción común del conocimiento científico. 


\section{Desde la dimensión procedimental:}

4. El personal docente tiene una MEDIANA actividad científica, en cuanto al número de publicaciones comprometidas en el Plan de Ciencia y Técnica y es MEDIANA su participación en eventos que evidencien el enriquecimiento científico de su desempeño integral, y evidencian MEDIANAMENTE tener destrezas lingüísticas, textuales, pragmáticas, tecnológicas y socioculturales en estas acciones dentro de un desempeño integral comunicativo.

Estas regularidades servirán de pauta para cumplimentar la fase de diseño de las acciones de superación que constituyen el objetivo del presente trabajo y su propuesta.

\section{Resultados y su análisis}

La propuesta de superación se rige por los siguientes objetivos generales:

- Contribuir a la profundización y sistematización delos conocimientos teóricosy prácticos que posibilitan el eficiente desempeño científico-comunicativo del personal docente de los centros universitarios municipales sobre las bases teóricas y metodológicas de la formación de competencia comunicativa.

- Potenciar la competencia en comunicación de resultados científicos en el personal docente de los centros universitarios municipales como aspecto favorecedor de una elevada actividad científica individual e institucional.

- Propiciar un ambiente de interacción y reflexión colectiva en torno a la actividad científica y al desempeño comunicativo de un sujeto investigador que posibilite transferir la experiencia a otros escenarios de intercambio científico.

- Fortalecer valores éticos y morales del profesorado en los centros universitarios municipales en su relación con sus semejantes y el entorno a través de un aprendizaje colaborativo, de intercambio de experiencias y fundamentado en necesidades del contexto socio-económico del municipio y particularmente de la institución docente.

En una representación gráfica del proceso de superación profesional (ver Figura 1) se observa la interrelación de los componentes estructurales y funcionales del proceso de superación que tiene en cuenta el diagnóstico del estado de la competencia y el diseño del sistema de acciones a partir de una negociación conjunta con los profesorado-alumnado, sus necesidades de superación, sus expectativas de transformación con dicho proceso. Esto se encauza a través de los objetivos generales del proceso que, a su vez, se reflejan en cada objetivo formativo competencial dentro de las actividades del diplomado (con su módulo 1 y 2) y el entrenamiento. Estas son las formas organizativas del postgrado que permiten el tránsito 
doi: http://dx.doi.org/10.15359/ree.21-2.2

URL: http://www.una.ac.cr/educare

CORREO: educare@una.cr

de docentes desde un nivel básico (formativo-interactivo) hasta un nivel de profundización (comunicativo-creativo). También se muestra la relación del blog científico como herramienta tecnológica de apoyo didáctico en las actividades del proceso de superación.

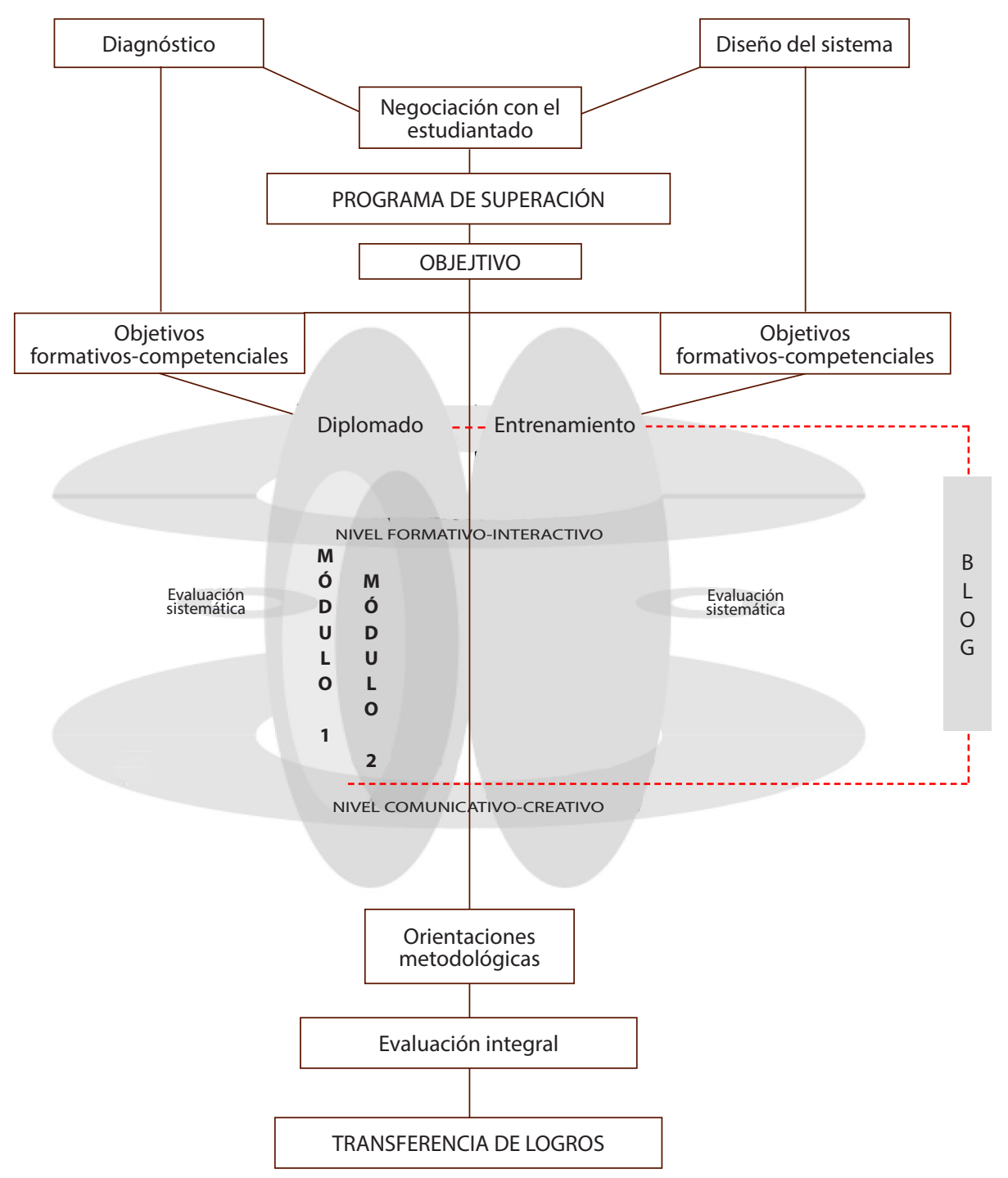

Figura 1: Representación gráfica del proceso de superación (elaboración propia).

Con la guía de los objetivos y la representación gráfica del proceso (ver Figura 1), se presenta la dosificación de los contenidos de las acciones de superación con el total de horas que incluye las horas presenciales y las horas de estudio independiente (ver tabla 1): 
Tabla 1: Dosificación de contenidos de acciones de superación

\begin{tabular}{llc}
\hline Tema & & Total de horas \\
\hline Diplomado. Módulo 1: Comunicación científica & 238 \\
\hline Tema I & Comunicación científica & 48 \\
Tema II & Gestión de publicación en la comunicación científica & 24 \\
Tema III & El texto científico & 100 \\
Tema IV & La comunicación de resultados científicos en la construcción del & 62 \\
& conocimiento. & 468 \\
\hline Diplomado. Módulo 2. Competencia comunicativa y comunicación de resultados científicos & 68 \\
\hline Tema V & La competencia comunicativa en el panorama de las competencias & 124 \\
& profesionales & 72 \\
Tema VI & La comunicación & 200 \\
Tema VII & La comunicación como proceso de participación y compartir experiencias. \\
Tema VIII & Talleres creativos & 115 \\
\hline Entrenamiento & & 40 \\
\hline Tema I & Producción de textos científicos & 25 \\
Tema II & Comunicación de resultados & 25 \\
\hline Tema III & Comunicación interactiva & \\
\hline
\end{tabular}

Nota: Elaboración propia.

Este programa se presentó a las autoridades competentes de la Universidad Central “Marta Abreu" de Las Villas para su correspondiente aprobación de implementación.

\section{Orientaciones metodológicas y organizativas}

La implementación de las actividades docentes debe combinar componentes de orden teórico que garanticen la adquisición de una base conceptual requerida, con los de orden práctico que posibilitarán el desarrollo de habilidades y destrezas desde el nivel de familiarización, pasando por el de reproducción hasta el más productivo. Para garantizar esta doble concepción de estructura de los contenidos, se utilizarán como formas de organización de la enseñanza: conferencias especializadas, talleres docentes, contenidas todas en un diplomado que a su vez se ha organizado en dos módulos. Luego de manera independiente se ha optado por un entrenamiento para completar así el programa de superación profesional. 
doi: http://dx.doi.org/10.15359/ree.21-2.2

URL: http://www.una.ac.cr/educare

CORREO: educare@una.cr

La conferencia especializada permite dar una visión panorámica del estado del arte de las áreas temáticas fundamentales, familiariza a sus participantes con esos contenidos; por lo que deben constituir una forma inicial de preparación de profesorado-alumnado para que puedan ir transitando por los niveles de dominio y facilitar un enfoque sistémico e integral, también interdisciplinario, en el avance del aprendizaje de los contenidos que en su unión tributan al concepto de competencia en la comunicación de resultados científicos, una suma de agregados conceptuales individuales. Optar inicialmente por la conferencia permite destacar lo esencial ante la gran cantidad de materiales y flujo de información al respecto.

Por su parte, el taller docente permite adentrarse en el valor práctico del dominio de los contenidos en un aprender-haciendo, desarrollando una actitud científica con valor interdisciplinario y un enfoque sistémico donde se establezca la relación de una tarea común entre quien forma-alumnado-alumnado. Ese trabajo grupal y el uso de métodos y técnicas adecuadas para el estímulo de la actividad productiva y creativa buscan la integración, en un solo proceso, de la docencia, la investigación y la práctica. Por ello, la dosificación del plan de superación contempla la integración de ambas formas organizativas para el desarrollo de cada temática y su sistema de contenidos.

Es necesario destacar que cada forma de organización debe salirse del marco tradicional en la que prevalezca el estímulo a la participación activa del profesorado-alumnado, el intercambio, la colaboración, la discusión y la reflexión, de ahí que se pretenda conjugar los métodos de niveles productivo y creativo, con elementos de una didáctica secuencial-competencial tan encaminada en otras experiencias formativas por competencias.

Tan es así que en cada conferencia especializada y taller docente cada formador o formadora partirá con un ejercicio creativo consistente en una tarea docente integradora en la que se marcará desde el inicio el nivel de independencia cognoscitiva hacia donde deberá llegar cada docente- estudiante una vez haya asimilado, de manera integrada, el contenido de la clase. La tarea docente integradora parte del objetivo formativo (la competencia a desarrollar) que guiará la solución del problema (esbozado en la tarea integradora) y a continuación los criterios de evaluación de desempeño para el dominio del contenido, que abarcan desde los niveles de familiarización, reproductivos-interpretativos, aplicativos, metacognitivos hasta el creativo, con el valor de la transferencia de los conocimientos, hábitos y habilidades alcanzados en el desarrollo de la actividad de aprendizaje.

La declaración del objetivo en forma de competencia reviste una importancia orientadora en el diseño de la tarea docente, y tendrá la siguiente estructura: 1-habilidad expresada en una acción (verbo)+ el objeto de competencia (vinculado al contenido)+ condición de la competencia expresada en la actividad o tarea (vinculado a su nivel de profundidad, su nivel de asimilación (reproductivo, productivo o creativo), condiciones de estudio (método) y hasta puede declarar la intencionalidad educativa. 
Las tareas que se elaboren deben tener un carácter integrador porque precisan incluir situaciones que exijan a cada docente-estudiante utilizar conocimientos ya abordados de manera previa; y un carácter formativo porque conduce le conduce a asumir una posición ante el fenómeno en estudio. Este carácter formativo que acompaña la integración cumple una función educativa y desarrolladora que ofrece al sujeto aprendiz la posibilidad de comprender la unidad material del mundo y los métodos para afrontar y resolver los problemas de la cotidianidad.

La integración de los contenidos contempla tres aristas: lo académico, manifestado por el desarrollo de conocimientos y habilidades para generar modos de actuación propios; lo laboral, concebido a partir de actividades conformes con la profesión, apoyado en la vinculación del estudio con el trabajo; y lo investigativo, a través de técnicas y métodos propios de la actividad científico-investigativa que refuercen lo académico y lo laboral.

En esta didáctica integradora, la tarea docente, enunciada desde el inicio, es referencia constante para la actividad de aprendizaje, cualquiera que sea la forma de organización: en la fase introductoria o de apertura es meta para la activación de los conocimientos previos, la disposición al aprendizaje de los nuevos contenidos dados por una reflexión sobre la pertinencia de ellos; luego el tratamiento de los nuevos contenidos conjugando los conocimientos, habilidades y actitudes que lo conforman, para potenciar la autorreflexión del alumnado respecto a la asimilación de ese contenido. El desarrollo concibe la preparación del profesorado-alumnado para la transferencia de esos conocimientos, habilidades y actitudes hacia el desempeño, que se enmarca en la actividad propiamente enfrentada por cada alumno o alumna con la guía de la tarea docente integradora y los niveles de ayuda que le ofrece el profesorado, tanto de manera individual como grupal y que se denomina práctica guiada. Aquí cada docente que orienta en la planeación didáctica para este momento utilizará métodos oportunos que estimulen la actividad productiva a través de acciones que conduzcan al dominio del contenido de la temática hasta su sistematización. En el momento del cierre de la secuencia didáctica el profesorado-alumnado se enfrenta a la tarea integradora reflejada en su desempeño como resultado y al cual ha llegado con una autovaloración (indicador metacognitivo) de los niveles de logros, de acuerdo con los criterios de medidas, por tanto, no es una evaluación guiada solo por el docente en su función orientadora, aun cuando es la figura que direcciona el proceso de enseñanza-aprendizaje. En esta fase final cada docente-estudiante recibirá nuevas orientaciones para la transferencia de su aprendizaje al nivel de desempeños hacia otros contextos y situaciones que demanden la solución de otros problemas a través de la integración de saberes teóricos y prácticos con independencia creativa.

La organización del plan de acciones de superación a través de un diplomado con dos módulos y de manera independiente un entrenamiento, se fundamenta en las necesidades de aprendizaje establecidas en la fase de diagnóstico inicial y el criterio de un mejor acercamiento a las áreas disciplinares que integran la variable de trabajo competencia en la comunicación de resultados científicos. De tal manera se propone comenzar por temáticas relacionadas en el primer módulo sobre comunicación científica y el segundo módulo sobre competencia comunicativa. 
doi: http://dx.doi.org/10.15359/ree.21-2.2

URL: http://www.una.ac.cr/educare

CORREO: educare@una.cr

En el primer módulo, el docente-estudiante adquiere los contenidos fundamentales de la actividad de comunicación científica desde su conceptualización y enfoques, pasando por su variedad de géneros discursivos, las normas de redacción y estilo, el aspecto productivo de textos hasta el pragmático de gestión de la publicación para redondear todo ello finalmente en la actividad integral de comunicación de resultados científicos dentro de la cadena de construcción del conocimiento científico.

Por su parte, el segundo módulo ya se acerca a la visión competencial de esa actividad productiva y comienza estableciendo las bases teóricas de la competencia comunicativa, luego profundiza en la comunicación como proceso de mediación social y desde el enfoque ritual expresivo de verla como un compartir experiencias, en este caso científicas e investigativas. Finalmente, el módulo cierra con talleres creativos que se centran en potenciar recursos personológicos de interacción social del alumnado de acuerdo con la concepción de competencia como configuración psicológica compleja que regula su desempeño eficiente.

Con el entrenamiento el grupo de docentes-estudiantes perfeccionará sus destrezas adquiridas con la intención de actualizarlas en tres dimensiones: la productiva, la comunicativa y la interactiva, donde ya se enfrentarán a demandas reales o simuladas de actividades de comunicación científica y en contextos más complejos.

En la planeación didáctica se utilizarán métodos productivos y creativos en pos de lograr el desarrollo del pensamiento, la imaginación, la participación, la activación del conocimiento y la independencia cognoscitiva, el involucramiento, la influencia mutua para la asimilación de conocimientos, desarrollo de habilidades, actitudes y valores siempre muy vinculados a las tareas que deben llevar a cabo para que se propicie la calidad del aprendizaje, el autoaprendizaje, el autoperfeccionamiento y autodirección del grupo. Para ello se emplearán los procedimientos y técnicas más adecuados a cada situación como: discusión grupal, estudio de casos, formulación de problemas, elaboración de preguntas, arribo a conclusiones, búsqueda de ejemplos, el diálogo, el debate, dramatizaciones / simulaciones, entre otros.

Los medios de enseñanza que se utilicen deben crear las condiciones materiales favorables para cumplir con las exigencias científicas durante el proceso de enseñanza aprendizaje, hacer más objetivos los contenidos de cada dirección de estudio y, por tanto, lograr mayor eficiencia en el proceso de asimilación del conocimiento, creando las condiciones para el desarrollo de capacidades, hábitos, habilidades y la formación de convicciones. De acuerdo con los métodos utilizados, son fundamentales los materiales bibliográficos, el uso de computadoras y conexión a internet para acceder a la blogosfera, pancartas, papelógrafos, etc.

Cada docente orientador $\mathrm{u}$ orientadora deberá ir evaluando diferentes momentos del proceso, teniendo en cuenta la concepción de las direcciones de superación para lo cual podrá utilizar la evaluación diagnóstica fundamentalmente en una fase inicial y la evaluación formativa 
para el desarrollo del proceso, para lo cual se apoyará en la autoevaluación, la coevaluación y la heteroevaluación. En su condición de evaluación formativa y adoptando la variante didáctica de orientar cada actividad a partir de un objetivo formulado como competencia a desarrollar, se evidencia el carácter sistemático toda vez que los indicadores de evaluación de desempeño constituyen, en sí mismos, herramientas para la autoevaluación de los niveles de logros del alumnado, además de la evaluación del profesorado orientador en cada actividad docente. Por su parte, el programa incluye evaluaciones parciales al culminar cada módulo donde se integrarán los contenidos, el cumplimiento de las tareas integradoras y cada alumno o alumna completará la evaluación, primero con la producción de un texto científico y en el segundo módulo expondrá oralmente los resultados ya recogidos en el texto evaluado y perfeccionado estructural y metodológicamente para ese entonces, por lo que se recomienda que el género discursivo orientado sea factible para el desempeño en la comunicación oral, como es el caso de la ponencia.

Finalmente, el entrenamiento tendrá una evaluación sistemática en cada sesión de trabajo, con un seguimiento tutorial, serán oportunas la modalidad de autoevaluación y coevaluación, debido a que los entrenamientos se realizarán en dos grupos y alrededor de la utilización del blog científico como herramienta de apoyo a la interactividad y a la comunicación.

Todo el programa de superación tributará a la presentación de los trabajos en el Fórum Científico de Base, y como antesala del evento municipal, que a la vez constituye un auténtico espacio de comunicación de resultados científicos.

Los criterios de evaluación que se sigan girarán alrededor de los indicadores de la variable de trabajo competencia en la comunicación de resultados científicos, reunidos en las tres dimensiones declaradas: cognitivo-instrumental, afectivo-motivacional y procedimental.

Se considera que la modalidad semipresencial es más propicia, pues al mismo tiempo que favorece la autogestión del conocimiento por parte del alumnado y el estudio independiente, no descarta el aprovechamiento del tiempo presencial para el aprendizaje colaborativo y el fomento de una disposición a compartir experiencias, en este caso de aprendizaje y desarrollo.

Se sugiere que el tiempo se distribuya según las formas a utilizar y los días de encuentro presencial; las conferencias deben tener un máximo de 4 horas de duración por encuentro, mientras que los talleres tendrán un mayor número de horas de 4 a 8 horas para priorizar el aprendizaje desde la práctica. En cuanto al entrenamiento, se priorizará la modalidad presencial y en cada temática se utilizarán 8 horas distribuidas en dos encuentros de 4 horas. El mayor tiempo de la concepción general del programa lo deberá tener el trabajo independiente, donde cada docente-estudiante podrá elevar sus habilidades prácticas y destrezas al nivel más creativo, de acuerdo con su capacidad de transferencia de los contenidos hacia la solución de nuevos problemas de demanda competencial comunicativa. 
doi: http://dx.doi.org/10.15359/ree.21-2.2

URL: http://www.una.ac.cr/educare

CORREO: educare@una.cr

Este proceso que conllevará a un estado de transformación ascendente hacia un nivel de desarrollo adecuado de la competencia en comunicación de resultados científicos se muestra gráficamente a continuación (ver Figura 2) con los aspectos que debe lograr el docente estudiante durante el proceso de superación, desde un nivel básico de conocimientos y habilidades sobre la comunicación científica, hasta un nivel de profundización expresada en niveles de dominio como destrezas. En ello se utiliza, como referente, la integración de los indicadores de competencia declarados anteriormente.

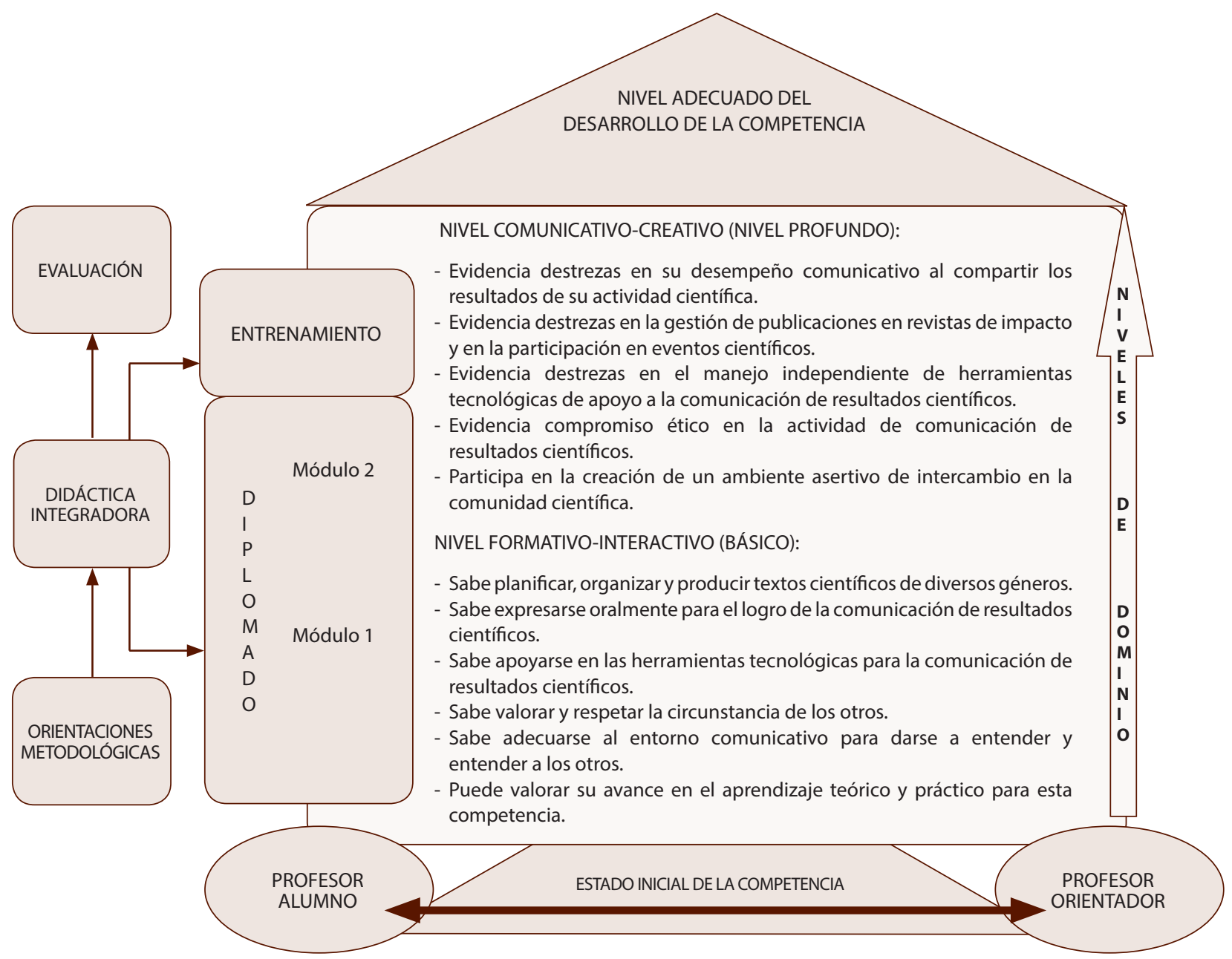

Figura 2: Representación gráfica del proceso de transformación de la superación a través del ascenso en los niveles de dominio de la competencia (elaboración propia). 


\section{Conclusiones}

Vista como proceso de formación continua a lo largo de la vida, la superación profesional es el eslabón superior de la formación permanente y en el contexto de la educación superior es la posibilidad para que la universidad pueda cumplir con su encargo social a través de tareas académicas, laborales e investigativas con profesoras y profesores preparados para resolver, de manera competente, los problemas de su entorno en sus modos de actuación profesional.

La consolidación de la experiencia de la municipalización, como parte de la estrategia de universalización de la educación superior en Cuba, ha potenciado la enseñanza de postgrado (en su modalidad de superación profesional), con los auspicios de la agenda priorizada educación superiordesarrollo local, en una concepción de desarrollo social basado en el conocimiento y la innovación.

Desde la cosmovisión de una formación para la nueva ciudadanía del siglo XXI, se va vertebrando, con mayor acogida entre la comunidad científica cubana, la educación de competencias, sobre todo en la enseñanza de postgrado, con el objetivo de contar en la sociedad con profesionales que actúen independiente y creativamente, y con motivación para resolver con ética problemas de su entorno.

La comunicación de resultados científicos como eslabón necesario en la cadena de construcción del conocimiento demanda de una competencia comunicativa por la parte docente de los centros universitarios municipales.

Se considera la competencia en comunicación de resultados científicos como configuración psicológica compleja que, en unidad cognitiva, afectivo-motivacional y procedimental, se autorregula en el sujeto para su desempeño eficiente, al compartir resultados de su actividad científica y adecuarse a las exigencias del contexto comunicativo a través del conocimiento y manejo de recursos lingüísticos, textuales, pragmáticos, tecnológicos y socioculturales.

Elenfoqueconfiguracionalen la concepción delascompetenciasconduce aconsideraciones específicas relativas al diagnóstico, organización, diseño, ejecución y evaluación de las acciones de superación para contribuir al desarrollo de competencia que involucran una perspectiva dinámica en la concepción de todos los componentes de este proceso de enseñanza-aprendizaje.

Un acercamiento diagnóstico al estado inicial de la variable de trabajo, así como a sus aspectos condicionantes, arrojó que la actividad científico-investigativa no tiene la prioridad necesaria en el Centro Universitario Municipal de Sagua la Grande ni en el nivel institucional ni en el profesorado, lo cual obstaculiza cumplir con el encargo social de la educación superior.

El estado inicial de la competencia en comunicación de resultados científicos en el profesorado del Centro Universitario Municipal de Sagua la Grande está en un nivel medio, dado por la valoración puntual de los conocimientos, habilidades, capacidades, actitudes, motivaciones, y representaciones del profesorado muestreado acerca de esta actividad en su desempeño científico-investigativo. 
doi: http://dx.doi.org/10.15359/ree.21-2.2

URL: http://www.una.ac.cr/educare

CORREO: educare@una.cr

La superación profesional con la integración de formas novedosas del postgrado es una vía efectiva para llevar la variable de trabajo a un estadio superior y cuenta con una disposición favorable, tanto institucional como del profesorado del Centro Universitario Municipal de Sagua la Grande.

El diseño de un plan de acciones de superación profesional interrelacionadas de manera sistémica y con una didáctica integradora contribuirá a potenciar la competencia en comunicación de resultados científicos en docentes de los centros universitarios municipales.

\section{Referencias}

Abarca-Fernández, R. R. (2010). Necesidad de un currículo por competencias. En U. Malaspina (Coord.), VI Congreso Iberoamericano de Docencia Universitaria. La opción por la interdisciplinariedad. El estudiante como protagonista (pp. 1-15). Lima, Perú: Pontificia Universidad Católica del Perú. Recuperado de http://academicos.iems.edu.mx/cired/docs/ $\mathrm{tg} /$ macroacademiaquimica/Necesidad\%20del\%20curriculo\%20por\%20competencias AbarcaFernandez.pdf

Añorga-Morales, J. A. (2014). La educación avanzada y el mejoramiento profesional y humano. Varona, 58 19-31. La Habana. Recuperado de http://www.redalyc.org/articulo. oa?id=360634165003

Beltrán, F. (s. f.). Desarrollo de la competencia comunicativa. Recuperado de http://www. robertexto.com/archivo9/compet comunic2.htm

Castellanos, B., Fernández, A. M., Llivina, M. J., Arencibia, V. y Hernández, R. (2003). Capítulo 3 Una visión alternativa de la competencia para la investigación educativa. En B. Castellanos, A. M. Fernández, M. J. Llivina, V. Arencibia y R. Hernandez (Autores), Esquema conceptual, referencial y operativo (ECRO) sobre la investigación educativa (pp. 60-82). La Habana, Cuba: ISPEJV. Recuperado de http://karin.fq.uh.cu/ vladimar/cursos/\%23Did\%E1cticarrrr/ Libros\%20de\%20Temas\%20Pedag\%F3gicos/Libro\%20ECRO.pdf

Chan, M. E. (2000). Entre la tecnofobia y la tecnofilia: El desafío de una comunicación educativa. La tarea, 12, 70-75. Recuperado de http://www.latarea.com.mx/indices/indice12.htm

Concepción, J. A. (2010). ¿Cómo estimular el desarrollo de la competencia comunicativa en idioma inglés? EduMecentro, 2(3), 42-49. Recuperado de http://www.revedumecentro.sld. cu/index.php/edumc/article/view/91/186

De la Paz, E. (2011). La competencia sociocultural en la formación inicial del profesor de lenguas extranjeras: Un modelo didáctico para su desarrollo. Revista IPLAC, 6. Recuperado de http:// www.revista.iplac.rimed.cu/index.php?option=com content\&view=article\&id=494:lacompetencia-sociocultural-en-la-formacion-inicial\&catid=38\&ltemid=394 
De la Rosa, M. (2004). El desarrollo de competencias comunicativas: Uno de los principales retos en la Educación Superior a Distancia. Primer Congreso Virtual Latinoamericano de Educación a Distancia. UNAM, México. Recuperado de https://educrea.cl/el-desarrollo-de-competenciascomunicativas-uno-de-los-principales-retos-en-la-educacion-superior-a-distancia/

Del Castillo, O. y Rodríguez, J. L. (2015). Desarrollo de la competencia comunicativa profesional en idioma inglés en estudiantes de la carrera de Medicina. Revista IPLAC, 5, 103-111. Recuperado de http://www.revista.iplac.rimed.cu/index.php?option=com content\&vie $\underline{w}=$ article\&id=2701:desarrollo-de-la-competencia-comunicativa-profesional-en-idiomaingles-en-estudiantes-de-la-carrera-de-medicina-13-pag-103-111-pdf

Escudero, J. M. (2008). Las competencias profesionales y la formación universitaria: Posibilidades y riesgos. Redu. Revista de Docencia Universitaria, 6(2), 1-20. Recuperado de http:// tecnologiaedu.us.es $/ \mathrm{mec} 2011 / \mathrm{htm} / \mathrm{mas} / 3 / 31 / 37 . \mathrm{pdf}$

Estrada, V. y Benítez, F. (2006). La gestión del conocimiento en la nueva universidad cubana. Revista Pedagogía Universitaria, 11(2). Recuperado de http://cvi.mes.edu.cu/peduniv/ index.php/peduniv/article/download/361/351

Fraga, O. (2005). Estrategia de superación profesional para la preparación teórico-metodológica del docente en el contenido de la ética martiana (Tesis doctoral inédita). Universidad de Ciencias Pedagógica “Félix Varela", Santa Clara, Cuba.

Freire, P. (2004). Pedagogía de la autonomía: Saberes necesarios para la práctica educativa. Buenos Aires: Siglo XXI Editores.

García-Ribeaux, T. M. y Zaldívar, M. (2013). La integración. Una vía de superación de los docentes de la carrera Educación Laboral - Informática. Pedagogía Profesional, 11(4). Universidad de Ciencias Pedagógicas "Héctor A. Pineda Zaldívar". Santiago de Cuba. Recuperado de http://www.pedagogiaprofesional.rimed.cu/index.php/numeros-publicados/2013/105volumen-11-numero-4-octubre-diciembre-2013/462-la-integracion-una-via-desuperacion-de-los-docentes-de-la-carrera-educacion-laboral-informatica-m-sc-thaismaria-garcia-ribeaux-dr-c-margarita-zaldivar-arena

González, M. y Alonso, M. (2012). Metodología para la evaluación del impacto de la superación en el desempeño profesional pedagógico de los docentes en las filiales universitarias municipales. Revista Congreso Universidad, 1(1). Recuperado de http://www. congresouniversidad.cu/revista/index.php/congresouniversidad/article/view/22

González, M. y Ramírez, I. (2011). La formación de competencias profesionales: Un reto en los proyectos curriculares universitarios. Odiseo, Revista Electrónica de Pedagogía, 8 (16). Recuperado de http://www.odiseo.com.mx/articulos/formacion-competenciasprofesionales-las-universidades-reto-proyectos-curriculares-univers 
doi: http://dx.doi.org/10.15359/ree.21-2.2

URL: http://www.una.ac.cr/educare

CORREO: educare@una.cr

González, V. (2006). La formación de competencias profesionales en la universidad. Reflexiones y experiencias desde una perspectiva educativa. XXI. Revista de Educación, 8, 175-187. Recuperado de http://rabida.uhu.es/dspace/bitstream/handle/10272/2010/b15168074. pdf? sequence $=1$

Mariño, M. y Ortiz, E. (2011).La formación pedagógica de los estudiantes universitarios.Didasc@ lia: Didáctica y Educación, 2(2), 1-10. Recuperado de http://runachayecuador.com/refcale/ index.php/didascalia/article/viewFile/205/163

Martín-Barbero, J. (2003). De los medios a las mediaciones. Comunicación, cultura y hegemonía. Bogotá: Convenio Andrés Bello. Recuperado de http://perio.unlp.edu.ar/catedras/system/ files/de los medios a las mediaciones.pdf

Mesa, G. (2011). Estrategia de superación profesional para potenciar en los directivos la competencia comunicativa para negociar (Tesis doctoral). Universidad Central "Marta Abreu" de Las Villas, Santa Clara, Cuba.

Núñez, J. (2010). Conocimiento académico y sociedad. Ensayos sobre política universitaria de investigación y postgrado. La Habana: Editorial UH.

Núñez, J., Montalvo, L. F. y Pérez, I. (2006). La gestión del conocimiento, la ciencia, la tecnología y la innovación en la nueva universidad: Una aproximación conceptual. En D. Hernández, F. Benítez, Y. Sánchez y S. A. Manzano (Comps.), La nueva universidad cubana y su contribución a la universalización del conocimiento (pp. 5-20). La Habana: Editorial Félix Varela.

Ortiz, E. (2006). Fundamentos psicológicos del proceso educativo universitario. Universidad de Holguín Oscar Lucero Moya, Cuba. Recuperado de http://cvi.mes.edu.cu/redees/wpcontent/uploads/2012/12/Fundamento-psicologicos.pdf

Peralta, L. (2013). Programa de superación profesional para potenciar la competencia didáctica del profesor universitario (Tesis doctoral inédita). Universidad Central "Marta Abreu" de Las Villas. Santa Clara, Cuba.

Pla, R. (2002). Modelo del profesional de la educación para asumir las tendencias integradoras de la escuela contemporánea. La Habana: Universidad Pedagógica Ciego de Ávila.

Pompa, Y. y Pérez, I. A. (2015). La competencia comunicativa en la labor pedagógica. Revista Universidad y Sociedad, 7(2), 160-167. Recuperado de http://scielo.sld.cu/pdf/rus/v7n2/ rus22215.pdf

Posada, R. (2004). Formación superior basada en competencias: Interdisciplinariedad y trabajo autónomo del estudiante. Revista Iberoamericana de Educación. Recuperado en http:// www.rieoei.org/edu sup22.htm 
Recino, U., Quesada, A. M., Finalet, E. y González, M. F. (2014). Herramienta metodológica para desarrollar competencia comunicativa en idioma inglés en las universidades médicas cubanas. Edumecentro, 6(2), 214-230. Santa Clara. Recuperado de http://scielo.sld.cu/ scielo.php?script=sci arttext\&pid=\$2077-28742014000200015

Roméu, A. (2007). El enfoque cognitivo, comunicativo y sociocultural en la enseñanza de la lengua y la literatura. En A. Roméu, L. M. Sales, I. Domínguez, T. Pérez, X. Rodríguez, A. Toledo... F. Martín (Autores), El enfoque cognitivo comunicativo y sociocultural en la enseñanza de la lengua y la literatura (pp. 17-56). La Habana: Editorial Pueblo y Educción.

Ruíz, A. y Roque, C. Y. (2015). Superación profesional en competencias para la comunicación de resultados científicos y en pos del desarrollo local. Revista Argentina de Educación Superior, 7(10), 86-107. Recuperado de http://www.revistaraes.net/revistas/raes10 art5.pdf

Ruiz, M. (2010). Hacia una pedagogía de las competencias. México: Ediciones CICEP.

Tejeda, R. y Sánchez, P. R. (2012). La formación basada en competencias profesionales en los contextos universitarios. Recuperado de http://cvi.mes.edu.cu/redees/wp-content/ uploads/2012/12/La-formacion-basada-en-competencias.pdf

Tobón, S. (2005). Formación basada en competencias. Pensamiento complejo, diseño curricular y didáctica. Bogotá: Editorial ECOE.

Valdesprieto, M. y Delgado, L. (2012). Algunas consideraciones sobre la evaluación del impacto de la superación. Revista Varela. La Habana.

Zabalza, M. A. (2012). Las competencias en la formación del profesorado: De la teoría a las propuestas prácticas. Tendencias pedagógicas, 20, 1-32. Recuperado de https://dialnet. unirioja.es/descarga/articulo/4105027.pdf 